\title{
Numerical Simulation of Transient Electromagnetic Response of Unfavorable Geological Body in Tunnel
}

\author{
Lubo Meng ${ }^{1, a}$, Tianbin $\mathrm{Li}^{1, \mathrm{~b}}$, Zheng Duan ${ }^{2, \mathrm{c}}$ \\ ${ }^{1}$ State key Laboratory of Geohazard Prevention and Geoenviroment Protection, \\ Chengdu University of Technology, Sichuan Chengdu, 610059,China \\ ${ }^{2}$ Third Railway Survey and Design Institute Group Corporation Tianjin, China \\ a menglubo@163.com, ${ }^{b}$ Itb@cdut.edu.cn, ${ }^{c} 147549330 @ q q . c o m$
}

\begin{abstract}
Keywords: Transient electromagnetic method; response; unfavorable geological body; numerical
\end{abstract} simulation

\begin{abstract}
To investigate the transient electromagnetic method of response characteristics in the tunnel geological prediction, the finite element numerical simulation of unfavorable geological body of different location, different resistivity sizes, different shapes, and different volume size were carried out by ANSYS finite element software. The results show that secondary electromagnetic field of different location of unfavorable geological body have same decay rate, when detection distance from $30 \mathrm{~m}$ to $70 \mathrm{~m}$, transient electromagnetic responses are strongest, followed distance from $10 \mathrm{~m}$ to $30 \mathrm{~m}$ and from $70 \mathrm{~m}$ to $90 \mathrm{~m}$. The shape, volume and resistivity of unfavorable geological body have strong influence on transient electromagnetic response, unfavorable geological body more sleek, the greater the volume and the smaller the resistivity of unfavorable geological body, the secondary electromagnetic field decay slower.
\end{abstract}

\section{Introduction}

Transient Electromagnetic Methods is an advanced detection method based on electromagnetic induction. The method was firstly used in the field of surface detection, and some experts ${ }^{[1 \sim 3]}$ in this field have researched on the working ways as well interpretative methods and have indicated that the effect does work according to abundant practical application. In recent years, Transient Electromagnetic Methods are applied in advanced geological forecast in tunnel, of which the interpretation is generally analyzed by the advanced tunnel geological forecast experts combing with interpretation map and the geological condition of the tunnel workface. Though some experts ${ }^{[4]}$ have improved the field working and the interpretation methods and have got some achievements, and some of them, such as H.Y.Yang ${ }^{[5]}$, have explore the transient electromagnetic method in tunnel, and analyze its response characteristics, but the regularities and response characteristics of unfavorable geological condition are not summarized and unified.

From forecasting unfavorable geological body in tunnel, according to typical transient electromagnetic numerical simulation, this paper explore the transient electromagnetic response characteristics of different conditions of unfavorable geological body in tunnel.

\section{The basic principle of Transient Electromagnetic Methods}

The Transient Electromagnetic Methods (TEM) is a kind of time domain method, using Laplace domain waveform electromagnetic pulse, with no grounding back to the front line, vortex generates in the geologic body under the excitation of the primary field, and the size depend on the conductive ability of the geological body, and if the conductive ability is strong then vortex is strong. After the primary field disappears, vortex can not disappear immediately, because there is a transition process (attenuation process), and the transition process produces a quadratic field to the attenuation of underground. The changes of the quadratic field will reflect the electrical conditions of underground 
medium by using receive coil to receive the quadratic field. According to different delay time in the case of measuring induction emf, we get the attenuation characteristics of quadratic field, thereby detecting various unfavorable geological targets.

The electromagnetic field is regarded as isotropic, linearity, then equation of Maxwell as follows $^{[4]}$ :

$$
\begin{aligned}
& \nabla \times \vec{E}=-\frac{\partial \overrightarrow{\mathrm{B}}}{\partial t}-\vec{J}_{m} \\
& \nabla \times \vec{H}=\frac{\partial \overrightarrow{\mathrm{D}}}{\partial t}+\vec{J}
\end{aligned}
$$

Among the items concerned, $\vec{E}$, whose unit is $\mathrm{V} / \mathrm{m}$, is the electric field intensity; $\vec{D}$, whose unit is $\mathrm{C} / \mathrm{m}^{2}$, is the dielectric flux density; $\vec{H}$, whose unit is $\mathrm{A} / \mathrm{m}$, is the magnetic intensity; $\vec{B}$, whose unit is $\mathrm{Wb} / \mathrm{m}^{2}$, is the magnetic flux density; $\vec{J}$, whose unit is $\mathrm{A} / \mathrm{m}^{2}$, is the electric current density; $\vec{J}_{m}$, whose unit is $\mathrm{V} / \mathrm{m}^{2}$, is the magnetic current density. The constitutive equations as follows ${ }^{[4]}$ :

$$
\begin{aligned}
& D=\varepsilon E \\
& B=\mu H \\
& J=\sigma E \\
& J_{m}=\sigma_{m} E
\end{aligned}
$$

Among the items concerned, $\varepsilon$, whose unit is $\mathrm{F} / \mathrm{m}$, is the dielectric constant; $\mu$, whose unit is $\mathrm{H} / \mathrm{m}$, is the permeance factor; $\sigma$, whose unit is $\mathrm{S} / \mathrm{m}$, is the electric conductivity; $\sigma_{m}$, whose unit is $\Omega / \mathrm{m}$, is the magnetic conductivity.

\section{The model scheme of numerical simulation}

The vertical length of mode was $200 \mathrm{~m}$ and horizontal length of model was $60 \mathrm{~m} \times 60 \mathrm{~m}$, cross-section shape of tunnel was semi-circular, radius was $8 \mathrm{~m}$ (Fig.1), The length of excavated tunnel was $30 \mathrm{~m}$. There were four types: air, exciting coil, wall rock and unfavorable geologic body, exciting coil was located in the center of wordface.

Numerical simulation scheme were shown in Table 1, the distance between unfavorable geological body and the working face was designed to $10 \mathrm{~m}, 30 \mathrm{~m}, 50 \mathrm{~m}, 70 \mathrm{~m}, 90 \mathrm{~m}$; shape of unfavorable geological body was designed for 4 types, respectively, the phere, cube, trapezoid bevel, cuboid; volume of unfavorable geological body was designed to $27 \mathrm{~m}^{3}, 64 \mathrm{~m}^{3}, 125 \mathrm{~m}^{3}, 216 \mathrm{~m}^{3}, 343 \mathrm{~m}^{3}$; resistivity of unfavorable geological body was designed to $1 \Omega \cdot \mathrm{m}, 10 \Omega \cdot \mathrm{m}, 50 \Omega \cdot \mathrm{m}, 100 \Omega \cdot \mathrm{m}, 200 \Omega \cdot \mathrm{m}$.

The model of transient electromagnetic field was created by ANSYS finite element software. It taked 3D- transient analysis method, SOLID117 unit was used to simulate element types. The geoelectric parameters of electromagnetic finite element model mainly contained permeability and resistivity, which were shown in Table 1 . The magnetic line parallel boundary is a suitable boundary condition in ANSYS, so it was taked as boundary condition in the model.

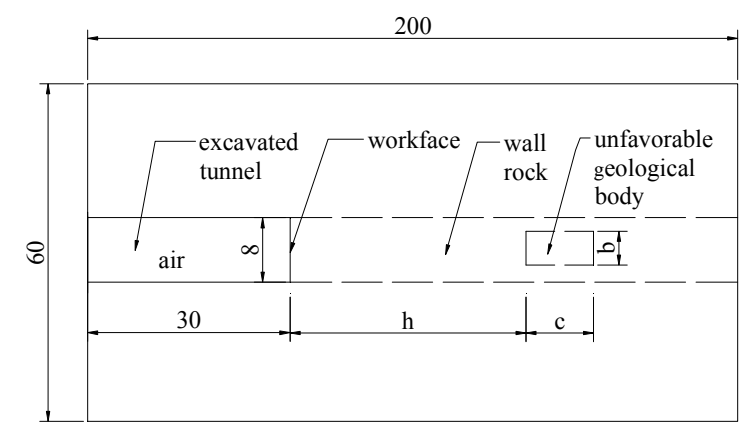

(a) skiagraph

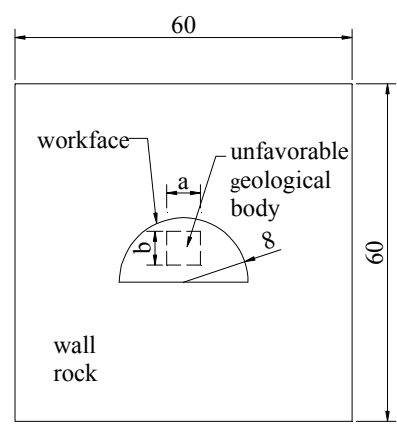

(b) cross section

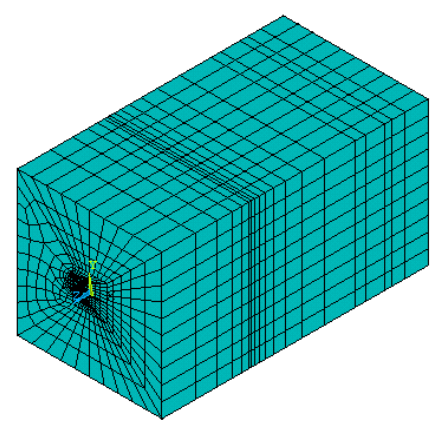

(c) mesh of model

Fig.1 Numerical simulation model (unit:m) 
The process of simulation: First, constant current was loaded at exciting coil elements; first electromagnetic field was created after enough time, and then cut down the current flow, current flow was changed between 0 to 1 , the calculation was stopped until satisfy certain time, a secondary induction field was formated. After simulation, the magnetic-field component of receiving coil was gotten, and then induced EMF curve can be calculated by integral.

Table1 The value of geoelectric parameters

\begin{tabular}{ccccccc}
\hline $\begin{array}{c}\text { distance/ } \\
\mathrm{h}(\mathrm{m})\end{array}$ & $\begin{array}{c}\text { shape of } \\
\text { unfavorable } \\
\text { geological body }\end{array}$ & $\begin{array}{c}\text { volume of } \\
\text { unfavorable } \\
\text { geological } \\
\text { body }\left(\mathrm{m}^{3}\right)\end{array}$ & $\begin{array}{c}\text { resistivity of } \\
\text { unfavorable } \\
\text { geological } \\
\text { body } / \rho(\Omega \cdot \mathrm{m})\end{array}$ & $\begin{array}{c}\text { resistivity } \\
\text { of wall } \\
\text { rock } \\
/ \rho(\Omega \cdot \mathrm{m})\end{array}$ & $\begin{array}{c}\text { resistivity } \\
\text { of coil } \\
/ \rho(\Omega \cdot \mathrm{m})\end{array}$ & $\begin{array}{c}\text { permeability } \\
/ \mu \mathrm{r}\end{array}$ \\
\hline 10 & sphere & 27 & 1 & 2000 & $1 \mathrm{e}^{-9}$ & 1 \\
30 & cube & 64 & 10 & 2000 & $1 \mathrm{e}^{-9}$ & 1 \\
50 & trapezoid bevel & 125 & 50 & 2000 & $1 \mathrm{e}^{-9}$ & 1 \\
70 & cuboid & 216 & 100 & 2000 & $1 \mathrm{e}^{-9}$ & 1 \\
90 & & 343 & 200 & 2000 & $1 \mathrm{e}^{-9}$ & 1 \\
\hline
\end{tabular}

\section{Analysis of simulation results}

\section{(1)Influence of distance}

When length of unfavorable geological body away from the working face is not the same, and other conditions are the same, the induced EMF changes with time at center of tunnel working face as shown in Fig.2, and where the vertical axis is logarithmic function of the induced EMF, horizontal axis is time. Fig.2 show that the induced EMF decays exponentially, after conversion of the logarithmic function, induced EMF - time curve parallel to each other on above different Detection range, and, at the same time, the order of induced EMF is $50 \mathrm{~m}>30 \mathrm{~m}>70 \mathrm{~m}>10 \mathrm{~m}>90 \mathrm{~m}$, this shows that the transient electromagnetic response characteristics significantly, when detection distance change from $30 \mathrm{~m}$ to $70 \mathrm{~m}$. This distance is suitable probing depth of the transient electromagnetic method in tunnel environment.

\section{(2)Influence of shape}

When shape of unfavorable geological body is not the same, and other conditions are the same, the induced EMF changes with time at center of tunnel working face as shown in Fig.3, and where the vertical axis is logarithmic function of the induced EMF. Fig. 3 show that the induced EMF decays exponentially, and at the same time, the order of induced EMF of different shape is sphere $>$ cube $>$ trapezoid bevel $>$ cuboid, this shows that unfavorable geological body more sleek, more obvious transient electromagnetic response, the slower decay, whereas, the faster decay.
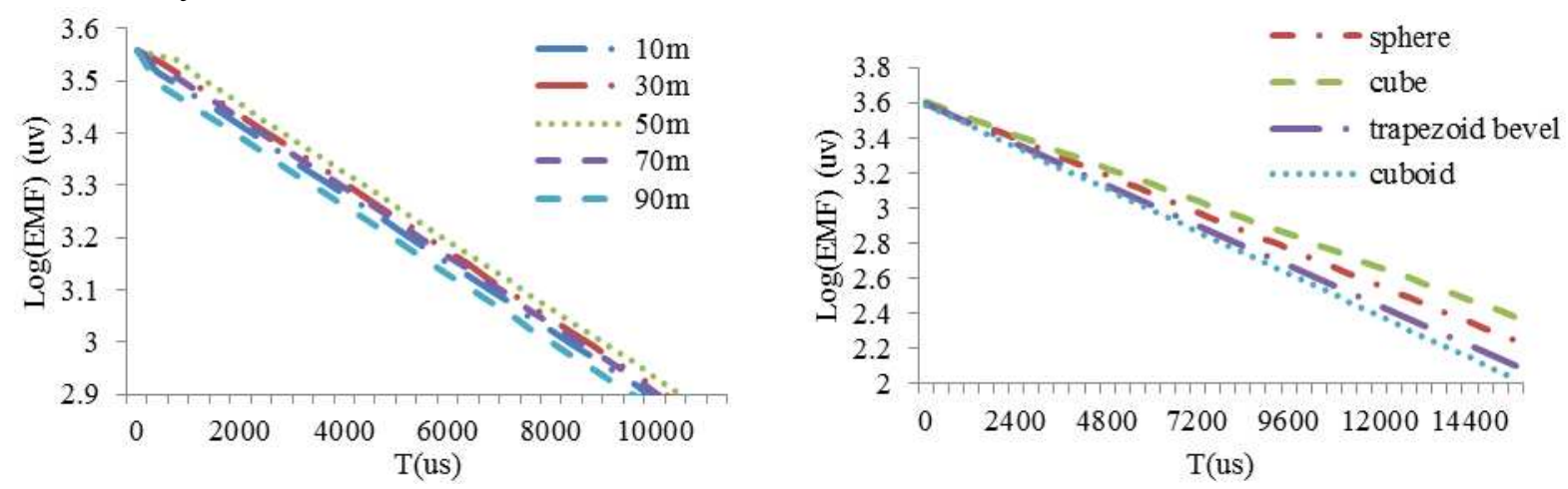

Fig.2 Induced EMF-time curve of different distance Fig.3 Induced EMF-time curve of different shape

\section{(3)Influence of volume}

When volume of unfavorable geological body is not the same, and other conditions are the same, the induced EMF changes with time at center of tunnel working face as shown in Fig.4, and where the vertical axis is logarithmic function of the induced EMF. Fig. 4 show that the induced EMF decays 
exponentially, and at the same time, the order of induced EMF is $343 \mathrm{~m}^{3}>216 \mathrm{~m}^{3}>125 \mathrm{~m}^{3}>64 \mathrm{~m}^{3}>$ $27 \mathrm{~m}^{3}$, this shows that the greater the volume of unfavorable geological body, the greater the induced voltage, the slower decay.

\section{(4)Influence of resistivity}

When resistivity of unfavorable geological body is not the same, and other conditions are the same, the induced EMF changes with time at center of tunnel working face as shown in Fig.5, and where the vertical axis is the induced EMF, horizontal axis is time. Fig.5 show that the induced EMF decays exponentially, and at the same time, the order of induced EMF is $1 \Omega \cdot \mathrm{m}>10 \Omega \cdot \mathrm{m}>50 \Omega \cdot \mathrm{m}>$ $100 \Omega \cdot \mathrm{m}>200 \Omega \cdot \mathrm{m}$, this shows that the smaller the resistivity of unfavorable geological body, the greater the induced voltage, decay more slowly, transient electromagnetic method is sensitive for low resistance.
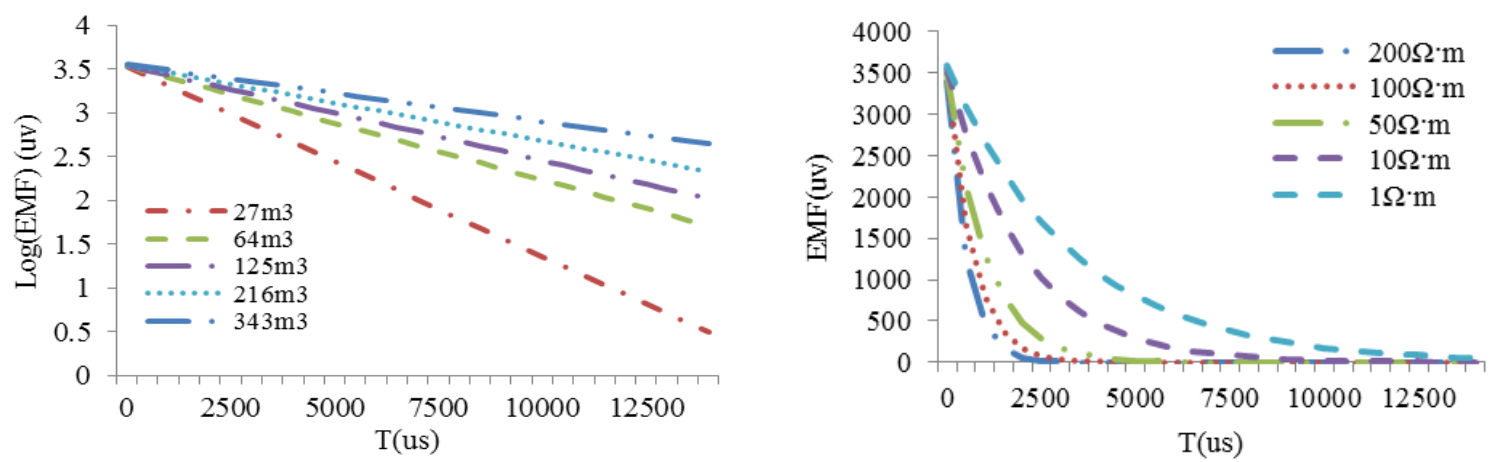

Fig.4 Induced EMF-time curve of different volume Fig.5 Induced EMF-time curve of different resistivity

\section{Conclusions}

By the analysis of numerical simulation of transient electromagnetism methods, understanding by the following:

(1) The location of unfavorable geological body has influence on transient electromagnetic response, secondary electromagnetic field of different location have same decay rate, when detection distance from $30 \mathrm{~m}$ to $70 \mathrm{~m}$, transient electromagnetic responses are strongest, followed distance from $10 \mathrm{~m}$ to $30 \mathrm{~m}$ and from $70 \mathrm{~m}$ to $90 \mathrm{~m}$, these be suitable probing depth of the transient electromagnetic method in tunnel environment.

(2) The shape of unfavorable geological body has influence on transient electromagnetic response, unfavorable geological body more sleek, secondary electromagnetic field decay slower.

(3) Volume and resistivity of unfavorable geological body are sensitive parameters, the greater the volume and the smaller the resistivity, the slower secondary electromagnetic field decay.

\section{Acknowledgements}

This work was supported by Key Program for Research Group of SKLGP (SKLGP2009Z002) and Open Fund Projects of SKLGP (GZ2007-06).

\section{References}

[1] B.R.Spies, P.D.Parke. Geophysics, Vo1.49, No.7 , 902-912 (1984).

[2] B.Y.JIANG. Practical near zone transient electromagnetic methods exploration. BeiJin:Geology Publishing House(1998) (in Chinese).

[3] H.R.PO. Theory of electromagnetic depth sounder methods. BeiJin:Geology Publishing House(1990) (in Chinese).

[4] X.Li. Theory and Apply of Transient Electromagnetic Depth Sounder Methods, XiAn:ShanXi Science and Technology Publishing House(2002) (in Chinese).

[5] H.Y.Yang, J.H.Yue. Journal of Jilin University, (1):129-134(2008) (in Chinese). 